\title{
CLIMATE-SMART CONSERVATION, A NEW WAY TO TACKLE THE GLOBAL SPECIES CONSERVATION CRISIS
}

\author{
SOŇA VA ŘACHOVÁ $1,2, *$ and BIKRAM SHRESTHA ${ }^{1,2}$
}

\author{
${ }^{1}$ Department of Biodiversity Research, Global Change Research Institute of the Czech Academy of Sciences, Bělidla 986/4a, 603 00 \\ Brno, Czech Republic \\ ${ }^{2}$ Institute of Environmental Studies, Charles University, Benátská 2, 12801 Prague 2, Czech Republic \\ *Corresponding author: varachova.s@czechglobe.cz
}

\section{ABSTRACT}

Climate change is something no one can ignore. While some people are still questioning the source of this issue, many are already working on solutions for the world's species, for which climate change might mean another step toward extinction. We are presenting here the basic idea of an innovative conservation approach, climate-smart conservation, which has a potential to mitigate the impacts of climate change and therefore protect some vulnerable species from demise. Next to its key characteristics we present examples of already ongoing practices involving climate-smart conservation and possible use of this approach in conservation of the snow leopard.

Keywords: biodiversity; climate change; climate-smart conservation; conservation; snow leopard

\section{Introduction}

Climate change represents a phenomenon of our time, and together with its possible impacts it has become a widely discussed topic (Bílá and Kindlmann 2019). Although there are some people still questioning or even denying the amount of human impact on this global matter, only humans are capable of tackling this major issue. Unfortunately, impacts of climate change drastically affect a variety of species of both flora and fauna, and therefore they are negatively contributing to an already massive biodiversity loss. There are many countries and societies realizing the current biodiversity crisis and they are also trying to face this problem. However, their conservation plans are often built on traditional approaches and are lacking innovation for a fast adaptation when facing unpredictable effects of climate change. The key to this can be a modification of such approaches, as for example an adaptive management based on an idea of an implement-monitor-evaluate-adjust cycle, which allows a later reaction to an at a first sight unexpected scenario (Hansen et al. 2010). Climate-smart conservation offers such innovation model through its focus on flexibility and consideration of a wide scale of scenarios (Stein et al. 2014), which together with prevision (Hansen et al. 2010) are necessary to overcome the possible changes in different regions coming with climate change. Its strategy can also be implemented into already planned, or even ongoing projects, which saves not only time but also money (Stein et al. 2014). This makes climate-smart conservation budget friendly and therefore more accessible for even smaller projects with less finances.

\section{Climate-Smart Conservation}

Climate-smart conservation is a new concept of an adaptive management, which seems to be able to over- come static habits in conservation. Its main idea is being explained by a couple of definitions throughout the literature and conservation organisations, and each of them usually presents its own key elements or characteristics to better define the main idea of this adaptation strategy. However, the main idea remains the same. The most complex description of this adaptation strategy was presented by Stein et al. (2014), who describe climate-smart conservation as:

"The intentional and deliberate consideration of climate change in natural resource management, realized through adopting forward-looking goals and explicitly linking strategies to key climate impacts and vulnerabilities."

Apart from this definition, Stein et al. (2014) also set their own key characteristics, closer identifying proper practice. First of them is Linking actions to Climate Impacts. Here Stein et al. (2014) explain that action plans and strategies should target existing threats alongside with the impacts of climate change. In case of including climate-smart conservation into already ongoing projects, an understanding, or a hypothesis of climate-related vulnerabilities reduction, or - on the other hand - a knowledge of any climate-related opportunities is necessary. The second, Embracing of Forward-Looking Goals, then explains that while facing the climate change, we can no longer rely on the traditional natural resources management approach, as it is often counting with static information about the environment, such as weather, habitats or species (Stein et al. 2014). New goals in conservation should focus on future conditions and they should consider possible need of strategies transitions. Another key element of climate-smart conservation described by Stein et al. (2014) is Consideration of Broader Landscape Context. This characteristic describes two important points in this climate change adaptation strategy: consideration of changes in distribution of species due to their adapta- 
tion to new conditions coming alongside climate change, and collaboration among institutions, communities or even landowners to support a common goal and avoid any conflicts in conservation actions and objectives. The key characteristic Adopting Strategies Robust to Uncertainty then explains that due to the uncertainties not only in the situations coming alongside the climate change, but also in the human reactions to it, new conservation strategies should benefit several future scenarios (Stein et al. 2014). Another vital element is then Employment of Agile and Informed Management, as it is required to deal with rapid changes of conditions not only related to the climate and the environment, but also to socioeconomics (Stein et al. 2014). Surely, Minimizing of Carbon Footprint is also important point, as strategies dealing with the climate change impacts should not contribute to this issue. Therefore, climate-smart conservation projects should direct their plans to minimize greenhouse gas emissions and the use of energy. The climate-smart projects should also Take into Account the Climate Influence on Project Success, as they should avoid investing into plans vulnerable to the climate change. Such vulnerability can appear in many sectors, including socioeconomics or ecology (Stein et al. 2014). Already during planning of the adaption strategy, Safeguarding of People and Nature should be considered as well, as focus directed not only on the ecosystem, but also on local communities contributes to a support by locals, which leads to a better success of the entire plan. Finally, climate-smart conservation should always focus on Avoiding of Maladaptation, as no strategy should compromise other climate change focused plans or disturb higher conservation targets (Stein et al. 2014).

The WWF (2021), which also works with the climate-smart conservation idea, comes with much simpler key elements:

a) Understanding the implications of climate change, including how human responses might lead to changes in other conventional threats.

b) Developing and implementing no-regret actions that address current threats, do not erode options for responding to future climate change, and avoid contributing to greenhouse gas emissions.

c) Taking an integrated approach to adaptation, contributing to nature conservation and fair, equitable and sustainable development.

d) Active learning to build capacity and work collaboratively to plan and respond to increasing change and uncertainty.

e) Bringing about changes in policy that create an enabling environment across scales (local to international) for adaptive governance.

However, many of their features are included in the Stein et al. (2014) key characteristics as well. WWF (2021) also offers its own description, which again is much more simplified: "Climate-smart conservation considers how climate and non-climate related pressures affect species, ecosystems and people."
Hansen et al. (2010) then comes with their own key elements (tenets) for the climate-smart conservation strategy. They consider important to include Adequate and appropriate space, which can later serve either as climate refugia with less severe climatic changes, as corridors to allow movement of species, or as network for allowing population connectivity. According to Hansen et al. (2010), it is also important to Reduce Non-climate stresses as "already stressed ecosystems and organisms are less resilient to climate-change effects". Another important tenet is then to Adopt Adaptive Management, which requires systematic evaluation of implemented strategies and in case appropriate adjustment. As Hansen et al. (2010) mention, "adaptation is a bicycle we must build while we ride it". The last tenet mentioned by Hansen et al. (2010) is to Reduce the Rate and Extent of Climate Change, which they emphasize is the key to conservation success as extension of climate change and its impacts can increase the adaptation costs but on the other hand decrease the chances of success. While all three sources present their key characteristics in a different way, they all share the same idea.

\section{Climate-Smart Conservation in the World}

To properly understand the right meaning of climate-smart conservation, it is better to present it in real examples. The first example of climate-smart conservation project is creation of setbacks with the Bruun Model for Beach Recession to help to predict natural shifts of beaches, and therefore preserve nesting beaches of hawkbill turtles (Eretmochelys imbricata) in Barbados (Fish et al. 2008). Hansen et al. (2010) describe, how sea level rising caused by climate change threatens nesting areas of sea turtles. Together with their sex determination being dependent on temperature during incubation, possible demise of sea turtle species is tightly connected to the climate change (Janzen 1994; Hansen et al. 2010). However, early determination of adequate spaces behind current nesting beaches - setbacks, which will be protected from human development and included into local conservation plans, can contribute to natural shift of nesting beaches inland, which even has a potential to be more sustainable then armouring, both ecologically and economically (Hansen et al. 2010). Another positive aspect of inland shifting of nesting beaches is that incubation temperature can be lowered by vegetation shade, naturally occurring in the setbacks, and therefore preserve sex ratio balance (Janzen 1994; Hansen et al. 2010)

Another example set by Hansen et al. (2010) comes from the Sundarbans National Park (West Bengal, India), where conflicts between Bengal tigers (Panthera tigris tigris) and humans are likely to escalate. The reason for this is a threat to local mangrove forest and therefore to local islands, which serve as protected tiger habitats 
and as home to local communities. According to Colette et al. (2007), the global rise of sea level together with other stressors can cause destruction of three quarters of mangroves in Sundarbans by the end of the century. To protect local tigers, it is necessary to use appropriate GIS - based spatial analyses and identify and protect new islands, which can serve as potential tiger habitats, despite the fact that currently they might seem unimportant or undeveloped (Hansen et al. 2010).

\section{Case of Snow Leopard}

Apart from examples presented in this paper, taking place by the coastline, climate-smart conservation has a great potential to be used in the mountain areas as well. More specifically, in the Himalayan region of Nepal, in conservation of snow leopard. Local population of snow leopard is scattered across the entire country and in many cases the area of their movement also crosses the national boundaries. Climate-smart conservation can be used to protect their possibility of movement by protecting areas of future corridors, which are likely to shift due to the climate change, and therefore contribute to snow leopard's better resilience. However, to determine future corridors we need to estimate potential suitable habitats for snow leopards.

Snow leopards in Nepal currently find refuge within some designated conservation areas and national parks
(Fig. 1). Nevertheless, in the future this might simply not be enough, as they will be most likely forced to search for prey and habitats elsewhere.

Another region considering implementing climate-smart conservation into their conservation plans to protect local snow leopard population is Central Asia (Kyrgyzstan and Tajikistan). In this area, there is an ongoing programme of Vanishing Treasures, an UNEP (United Nations Environment Programme) project to protect iconic species (Vanishing Treasures 2021). Also, in this case, one of the conservation points, where they are using this adaptation strategy, is "Integrating climate-smart measures into conservation planning, including ecological connectivity measures that account for shifting and changing habitats and other changes as a result of climate change" (Vanishing Treasures 2021).

\section{Conclusions}

Relatively new adaptation strategy, climate-smart conservation, offers an approach, which can help the world's biodiversity and us to prepare for changes inevitably coming with the climate change. Despite still being described in various ways, with every author the core stays the same, and in every way, it is meant to be implemented in a wide range of projects to help navigate the conservation into the future. The future might not seem too bright for the biodiversity, but the entire life is about

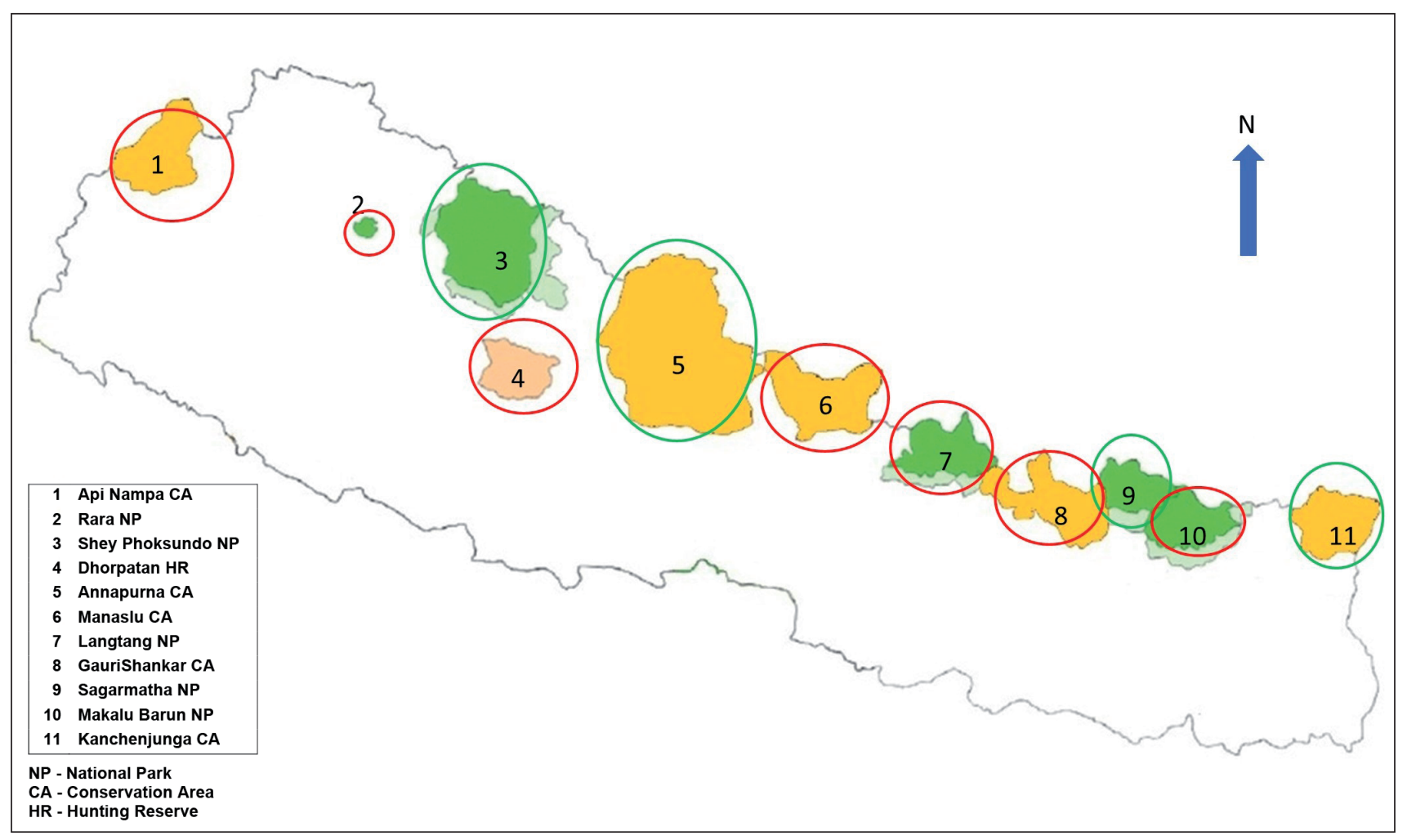

Fig. 1 Protected areas of Nepal in mountain range and possible snow leopard range: yellow colour - Conservation Area, dark green colour - National Park, pink colour - Hunting Reserve; green circle - area with snow leopard abundance/density data using genetic analysis or camera trap survey, red circle - area with no sufficient/rigorous data. 
adaptation, and we are right now standing at a tipping point, where we must decide how we want our future to look like and how we want our future generations to remember us. Climate change is happening and if species are able to adapt to a certain point, we are obliged to help them to make a step even further for them to be able to survive.

\section{Acknowledgements}

We thank Llion Myfyr for editing the language of the paper.

\section{REFERENCES}

Bílá K, Kindlmann P (2019) Is the Šumava National Park changing into a desert? A mini-review. Eur J Environ Sci 9: 72-76.
Colette A (2007) Case studies on climate change and world heritage. United Nations Educational, Scientific and Cultural Organization World Heritage Centre, Paris.

Fish MR, Côté IM, Horrocks JA, Mulligan B, Watkinson AR, Jones AP (2008) Construction setback regulations and sea-level rise: mitigating sea turtle nesting beach loss. Ocean Coast Manag 51: 330-341.

Hansen L, Hoffman J, Drews C, Mielbrecht E (2010) Designing climate-smart conservation: Guidance and case studies. Conserv Biol 24: 63-69.

Janzen FJ (1994) Vegetational cover predicts the sex ratio of hatchling turtles in natural nests. Ecology 75: 1593-1599.

Stein B, Glick P, Edelson N, Staudt A (2014) Climate-smart conservation: Putting adaptation principles into practice. National Wildlife Federation, Washington, D.C.

Vanishing Treasures (2021) Our work. https://vanishingtreasures .org/our-work/. Accessed 29 September 2021.

WWF (2021) Climate-smart conservation. https://wwf.panda.org /discover/our_focus/climate_and_energy_practice/what_we _do/climate_change_adaptation/climate_smart_conservation/. Accessed 29 September 2021. 